\title{
Novel Bipolar Bathophenanthroline Containing Hosts for Highly Efficient Phosphorescent OLEDs
}

\author{
Ziyi Ge, ${ }^{\dagger}$ Teruaki Hayakawa, ${ }^{\dagger}$ Shinji Ando,${ }^{\dagger}$ Mitsuru Ueda, ${ }^{\dagger}$ Toshiyuki Akiike, ${ }^{\ddagger}$ Hidetoshi Miyamoto, ${ }^{\ddagger}$ \\ Toru Kajita, ${ }^{\ddagger}$ and Masa-aki Kakimoto ${ }^{* \dagger}$ \\ ${ }^{\dagger}$ Department of Organic \& Polymeric Materials, Tokyo Institute of Technology, Ookayama 2-12-1, \\ Meguro-ku, Tokyo 152-8552, Japan and \\ ${ }^{*}$ Display Research Laboratories, JSR Corporation, 100, Kawajiri-cho,Yokkaichi, Mie 510-8552, Japan \\ mkakimot@o.cc.titech.ac.jp
}

\section{Supporting Information}

Contents Page

$\begin{array}{lll}\text { Calculation } & \text { Computational details } & \text { S-2 }\end{array}$

$\begin{array}{ll}\text { Measurements } & \text { S-2 }\end{array}$

Fabrication of OLEDs $\quad$ S-2

$\begin{array}{lll}\text { Table S-1 Theoretical and experimental energy levels. } & \text { S-3 }\end{array}$

Figure S-1 3D structure of TPAP, BCZBP, Me-BCZBP and m-BPABP $\quad$ S-3

Synthesis Experimental procedures for synthesis and spectroscopic characterizations $\quad$ S-4 6

${ }^{1} \mathrm{H},{ }^{13} \mathrm{C}$ NMR spectra $\quad$ S-6 8 
Computational Details. The calculated values of HOMO and LUMO energy levels and the triplet energy gaps $\Delta E\left(T_{1}-S_{0}\right)$ are evaluated by the DFT level of theory with the three-parameter Becke-style hybrid functional (B3LYP), in which the Becke exchange and LYP correlation functionals was adopted in conjunction with the Gaussian basis sets. The 6-31G(d) basis set was used for geometry optimizations of compounds under no constraints, and the $6-311 \mathrm{G}(\mathrm{d}, \mathrm{p})$ was used for calculations of molecular orbital energies (HOMO and LUMO) and total energies of molecules. A value of $\Delta E\left(T_{1}-S_{0}\right)$ was calculated as a difference between the total energies at the first excited triplet state $\left(T_{1}\right)$ and the ground singlet state $\left(S_{0}\right)$. These values shown in Table S-1 and Figure S-1 were performed using the software package of Gaussian-03 (Rev.C02 and D01) installed on a Compaq Alpha server GS-320 in the Global science and computing center, Tokyo Institute of Technology.

Measurements. ${ }^{1} \mathrm{H}-\mathrm{NMR},{ }^{13} \mathrm{C}-\mathrm{NMR}$ and measurements were carried out by a JEOL JNM-AL $300 \mathrm{MHz}$ spectrometer in $\mathrm{CDCl}_{3}$, or DMSO-d $\mathrm{d}_{6}$. Matrix Assisted Laser Desorption Ionization Time of Flight Mass Spectrometry (MALDI-TOF-MS) was taken on a Shimazu AXIMA-CFR mass spectrometer. The spectrometer was equipped with nitrogen laser $(\lambda=337 \mathrm{~nm})$ and with pulsed ion extraction. The operation was performed at an accelerating potential of $20 \mathrm{kV}$ by a linear-positive ion mode. Samples for MALDI-TOF-MS were prepared by casting the matrix compound (dithranol) onto the slide. Column (flash) chromatography was performed using 32-63 um silica gel. Absorption spectra were measured on a HP-8453 Diode Array spectrometer using THF. Emission spectra were measured on an Aminco-Bowman Series 2 luminescence spectrometer. Electroluminescence spectra were obtained using a PTI QM-2001-4 spectrophotometer. Current-voltage characteristics of the OLEDs were measured using a HP4155A semiconductor parameter analyzer (Yokogawa Hewlett-Packard Tokyo). The luminance was simultaneously measured using a model 370 optometer (UDT instruments, Baltamore, MD).

The experimental values of HOMO levels were determined with a Riken AC-2 photoemission spectrometer (PES), and those of LUMO $\left(S_{1}\right)$ and $T_{1}$ levels were estimated from the UV and phosphorescent spectra.

Fabrication of OLEDs. We fabricated OLEDs using bathophenanthroline derivatives doped with $6 \%$ mole $\operatorname{Ir}(\mathrm{ppy})_{3}$ as the emissive layer. Indium-tin oxide (ITO)-coated glass substrates were cleaned sequentially in ultrasonic baths of a 2-propanol/deionized water (1:1 volume) mixture, toluene, deionized water, and acetone before it was dried and treated with UV ozone. An $20 \mathrm{~nm}$ poly(ethylenedioxythiophene) (PEDOT) was spin-coated onto the ITO substrate from the $1 \mathrm{wt} \%$ aqueous PEDOT solution and dried at $140{ }^{\circ} \mathrm{C}$ for $1 \mathrm{~h}$ under vacuum. A $70 \mathrm{~nm}$ thick layer of bathophenathroline derivatievs doped with $6 \%$ mole $\operatorname{Ir}(\text { ppy })_{3}$ was deposited under vacuum as the emitting layer. A $30 \mathrm{~nm}$ TPBI layer was evaporated onto the emitting layer as a hole blocking layer at $2 \times 10^{-6}$ Torr. A $20 \mathrm{~nm}$ thick Cs:BCP (1:1) layer was deposited followed by an $100 \mathrm{~nm}$ thick aluminum layer at $1 \times 10^{-6}$ Torr as the cathode. 
Table S-1. Theoretical and experimental energy levels

\begin{tabular}{cccc}
\hline \multirow{2}{*}{ Compound } & $\begin{array}{c}\text { HOMO/LUMO } \\
\Delta E_{\text {номо-Lомо }} / \mathrm{eV}\end{array}$ & $\begin{array}{c}\mathrm{HOMO} / \mathrm{LUMO}_{\text {exp }} \\
\Delta E_{\text {номо-Lомо }} / \mathrm{eV}\end{array}$ & $\Delta E\left(T_{1}-S_{0}\right)_{\text {cal/exp }} / \mathrm{eV}$ \\
\hline CZBP & $5.48 / 2.19,3.29$ & $5.85 / 2.76,3.09$ & $2.68 / 2.41$ \\
m-CZBP & $5.57 / 2.22,3.35$ & $5.76 / 2.48,3.28$ & $2.77 / 2.61$ \\
BCZBP & $5.50 / 2.18,3.32$ & - & $2.70 /-$ \\
Me-BCZBP & $5.53 / 2.08,3.45$ & - & $2.77 /-$ \\
TPAP & $5.04 / 1.89,3.15$ & - & $2.54 /-$ \\
m-ТРAP & $5.16 / 1.99,3.17$ & $5.70 / 2.52,3.18$ & $2.71 / 2.52$ \\
BРАВP & $5.10 / 2.02,3.08$ & $5.51 / 2.62,2.89$ & $2.62 / 2.44$ \\
m-BРАВP & $5.14 / 1.98,3.16$ & - & $2.76 /-$ \\
\hline
\end{tabular}
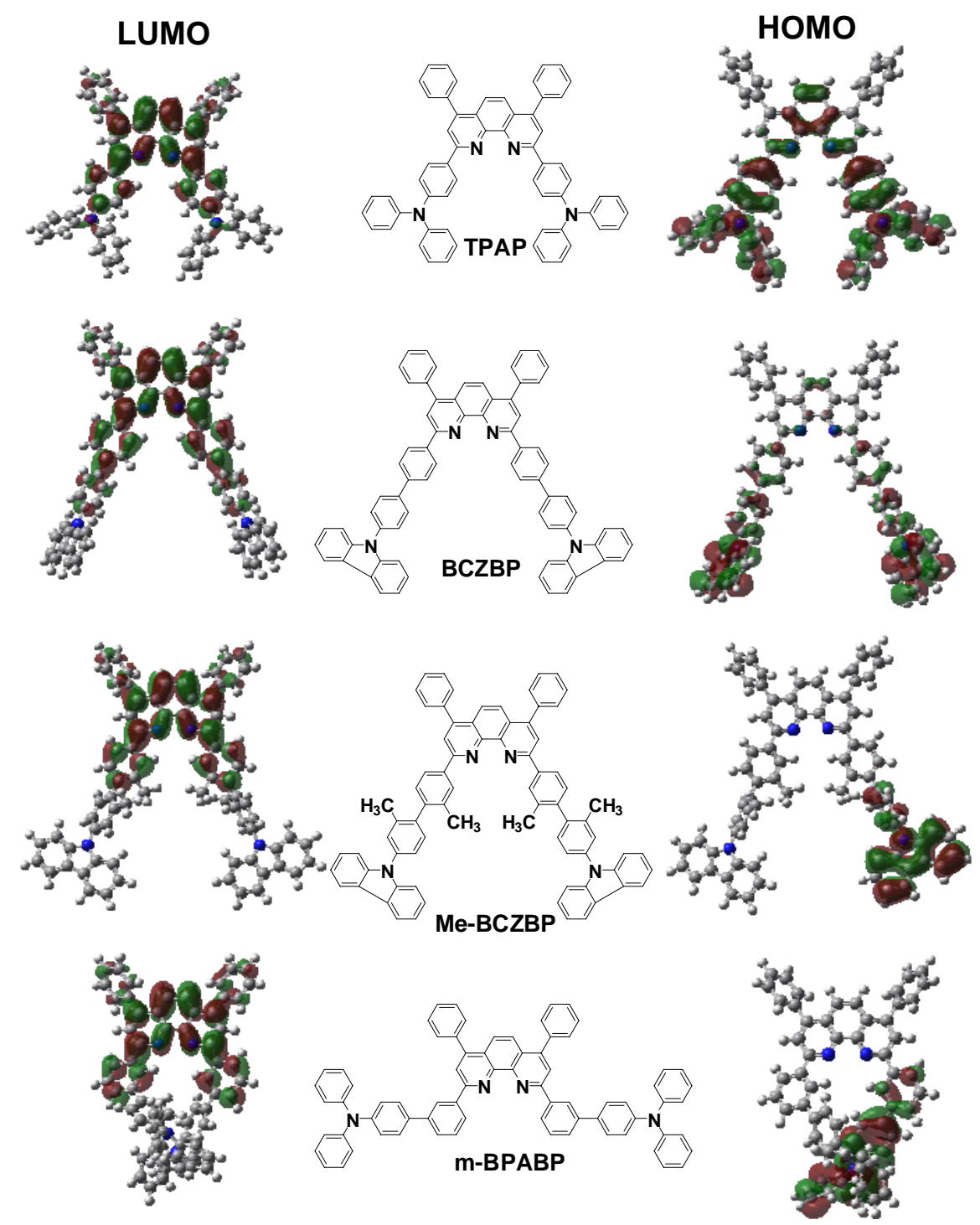

Figure S-1. 3D structure of TPAP, BCZBP, Me-BCZBP and m-BPABP 
The arylbromides, 9-(4-bromophenyl)carbazole (1), 9-(3-bromophenyl)carbazole (2), and 3-bromotriphenylamine (3) were prepared in the conditions according to the literatur. ${ }^{\mathrm{S}-1} 4^{\prime}-\mathrm{Bromo}-\mathrm{N}, \mathrm{N}-$ diphenylbiphenyl-4-amine (4) was prepared according to the method reported by Li et al. ${ }^{\mathrm{S}-2}$

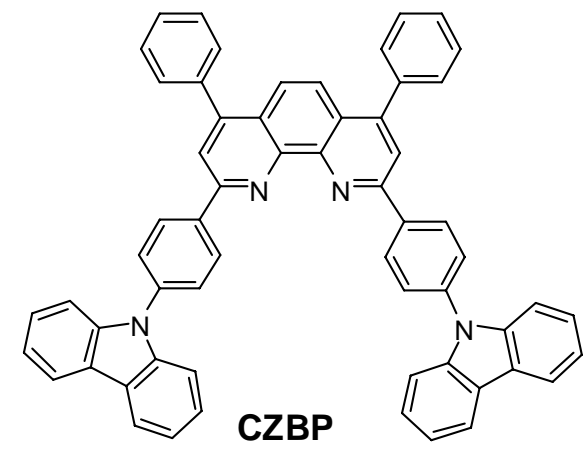

Synthesis of 2,9-bis(4-(9H-carbazol-9-yl)phenyl)-4,7-diphenyl-1,10-phenanthroline (CZBP). In a typical cross-coupling reaction, e.g., of CZBP, arylbromide 1 (1.160 g, $3.6 \mathrm{mmol})$ was dissolved in anhydrous ether $(80 \mathrm{~mL})$ in a $200 \mathrm{~mL}$ flask under argon at $0{ }^{\circ} \mathrm{C}$, then $n$-butyllithium $(1.52 \mathrm{~mL}, 4.0 \mathrm{mmol}$, 2.6 $\mathrm{M}$ in hexane) was added dropwise in $1 \mathrm{~h}$ and stirred for $1 \mathrm{~h}$. A suspension of bathophenanthroline $(0.500 \mathrm{~g}, 1.5 \mathrm{mmol})$ in anhydrous toluene $(20 \mathrm{~mL})$ was added. The mixture was heated at reflux for $24 \mathrm{~h}$, quenched with water and then extracted with dichloromethane. The organic layer was stirred with activated $\mathrm{MnO}_{2}(10.000 \mathrm{~g})$ for $6 \mathrm{~h}$. The mixture was dried over $\mathrm{MgSO}_{4}$ and then filtered. After removing the solvent the residue was purified by column chromatography on silica gel with dichloromethane as the eluent, which was then recrystallized from $\mathrm{CHCl}_{3}$ and hexane to give a white solid. Yield: 0.639 g, 52.3\%. M.p.: $219{ }^{\circ} \mathrm{C}$. ${ }^{1} \mathrm{H}$ NMR $\left(\mathrm{CDCl}_{3}, 300 \mathrm{MHz}, \mathrm{ppm}\right): 8.72-8.75$ (d, 4H), 8.19 (s, 2H), 8.13-8.16 (d, 4H), 7.89 (s, 2H), 7.79-7.82 (d, 4H), 7.52-7.67 (m, 14H), 7.40-7.46 (t, 4H), 7.26-7.31 (t, 4H). ${ }^{13} \mathrm{C}$ NMR $\left(\mathrm{CDCl}_{3}, 75 \mathrm{MHz}\right.$, ppm): 155.5, 149.6, 147.0, 140.8, 138.8, 138.7, 138.3, 129.8, 129.3, 128.7, 128.6, 127.4, 126.0, 123.9, 123.5, 120.8, 120.3, 120.1, 109.9. FT-IR $\left(\mathrm{KBr}, \mathrm{cm}^{-1}\right), 3055,1600,1563,1542,1480,1452,1382,1330$, 1311, 1235. MALDI-TOF mass (m/z): $815\left(\mathrm{M}^{+}\right)$. Elemental analysis calculated for $\mathrm{C}_{60} \mathrm{H}_{38} \mathrm{~N}_{4}: \mathrm{C}, 88.43 ; \mathrm{H}$, 4.70; N, 6.87. Found: C, 88.69; H, 4.63; N, 6.93.

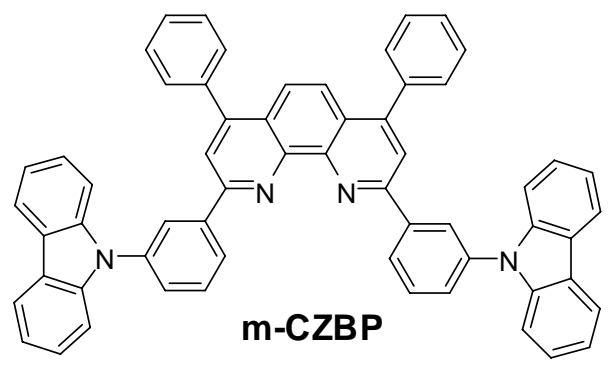

(S-1) Zhang, Q.; Chen, J.; Cheng, Y.; Wang, L.; Ma, D.; Jing, X.; Wang, F. J. Mater. Chem. 2004, 14, 895.

(S-2) Li, Z. H.; Wong, M. S.; Tao, Y.; D'Iorio, M. J. Org. Chem. 2004, 69, 921. 
Synthesis of 2,9-bis(3-(9H-carbazol-9-yl)phenyl)-4,7-diphenyl-1,10-phenanthroline (m-CZBP). $\mathrm{m}$-CZBP was prepared in the similar cross-coupling procedure starting from 2 and bathophenanthroline as a white solid. Yield: $43.0 \%$. M.p: $240{ }^{\circ} \mathrm{C} .{ }^{1} \mathrm{H}$ NMR $\left(\mathrm{CDCl}_{3}, 300 \mathrm{MHz}, \mathrm{ppm}\right)$ : 8.57-8.60 (d, 4H), 8.16-8.19 (d, 4H), 8.07 (s, 2H), 7.84 (s, 2H), 7.62-7.65 (d, 2H), 7.47-7.57 (m, 16H), 7.39-7.45 (t, 4H), 7.27-7.33 (t, 4H). ${ }^{13} \mathrm{C}$ NMR ( $\left.\mathrm{CDCl}_{3}, 75 \mathrm{MHz}, \mathrm{ppm}\right): 155.1,149.7,141.4,141.1,138.1,130.6,129.7,128.7,128.6$, 128.1, 126.9, 126.2, 126.1, 126.0, 123.9, 123.4, 120.5, 120.3, 119.9, 109.9. FT-IR (KBr, cm $\left.{ }^{-1}\right), 3053,1599$, 1560, 1540, 1477, 1452, 1375, 1334, 1314, 1230. MALDI-TOF mass (m/z): $815\left(\mathrm{M}^{+}\right)$. Elemental analysis calculated for $\mathrm{C}_{60} \mathrm{H}_{38} \mathrm{~N}_{4}$ : C, 88.43; H, 4.70; N, 6.87. Found: C, 88.26; H, 4.72; N, 6.75.

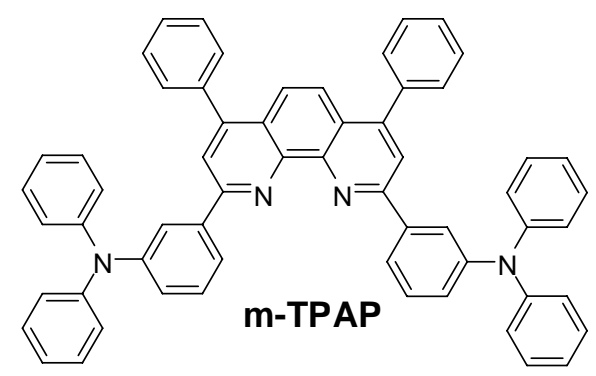

Synthesis of 3,3'-(4,7-diphenyl-1,10-phenanthroline-2,9-diyl)bis(N,N-diphenylaniline) (m-TPAP). $\mathrm{m}$-TPAP was prepared according to the general cross-coupling procedure using $\mathbf{3}$ and bathophenanthroline as a white solid. Yield: 51.2\%, M.p: $360{ }^{\circ} \mathrm{C} .{ }^{1} \mathrm{H}$ NMR $\left(\mathrm{CDCl}_{3}, 300 \mathrm{MHz}, \mathrm{ppm}\right)$ : 8.05-8.10 (d, 4H), 7.88 (s, 2H), 7.76 (s, 2H), 7.41-7.51 (m, 12H), 7.21-7.26 (m, 8H), 7.13-7.16 (m, 10H), 6.95-7.00 (t, 4H). ${ }^{13} \mathrm{C}$ NMR $\left(\mathrm{CDCl}_{3}, 75 \mathrm{MHz}, \mathrm{ppm}\right): 156.7,150.2,148.2,147.9,141.3,138.3,130.0,129.7,129.2,128.6,128.4,125.8$, 125.6, 124.2, 123.8, 123.6, 123.3, 122.5, 120.9. FT-IR $\left(\mathrm{KBr}, \mathrm{cm}^{-1}\right): 3056,1588,1492,1293$. MALDI-TOF mass $(\mathrm{m} / \mathrm{z}): 819\left(\mathrm{M}^{+}\right)$. Elemental analysis calculated for $\mathrm{C}_{60} \mathrm{H}_{42} \mathrm{~N}_{4}: \mathrm{C}, 87.99 ; \mathrm{H}, 5.17 ; \mathrm{N}, 6.84$. Found: $\mathrm{C}$, $87.76 ; \mathrm{H}, 5.14 ; \mathrm{N}, 6.79$.

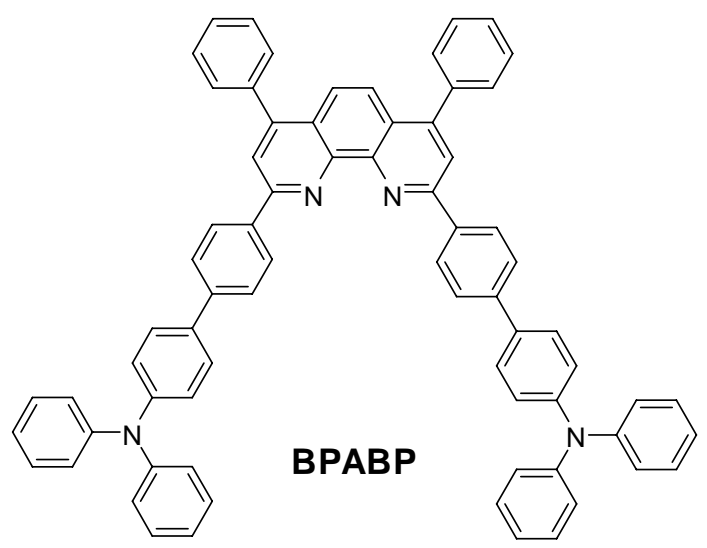

Synthesis of 4 ',4"-(4,7-diphenyl-1,10-phenanthroline-2,9-diyl)bis(N,N-diphenylbiphenyl-4-amine) 
(BPABP). BPABP was prepared according to the general cross-coupling procedure strating from $\mathbf{4}$ and bathophenanthroline as a yellow solid. Yield: $40.5 \%$, M.p: $300{ }^{\circ} \mathrm{C} .{ }^{1} \mathrm{H}$ NMR $\left(\mathrm{CDCl}_{3}, 300 \mathrm{MHz}, \mathrm{ppm}\right)$ : 8.58-8.60 (d, 4H), 8.13 (s, 2H), 7.80-7.83 (m, 6H), 7.50-7.62 (m, 14H), 7.25-7.30 (m, 8H), 7.12-7.18 (m,

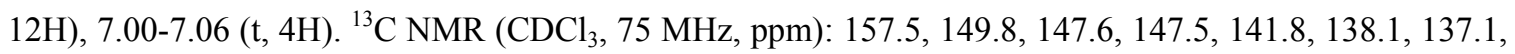
137.7, 134.2, 129.7, 129.2, 128.7, 128.6, 128.2, 127.7, 126.9, 125.9, 124.4, 123.7, 123.6, 123.0, 120.8 . FT-IR $\left(\mathrm{KBr}, \mathrm{cm}^{-1}\right)$ : 3054, 1590, 1490, 1295. MALDI-TOF mass $(\mathrm{m} / \mathrm{z}): 971\left(\mathrm{M}^{+}\right)$. Elemental analysis calculated for $\mathrm{C}_{72} \mathrm{H}_{50} \mathrm{~N}_{4}$ : C, 89.04; H, 5.19; N, 5.77. Found: C, 89.26; H, 5.04; N, 5.83.
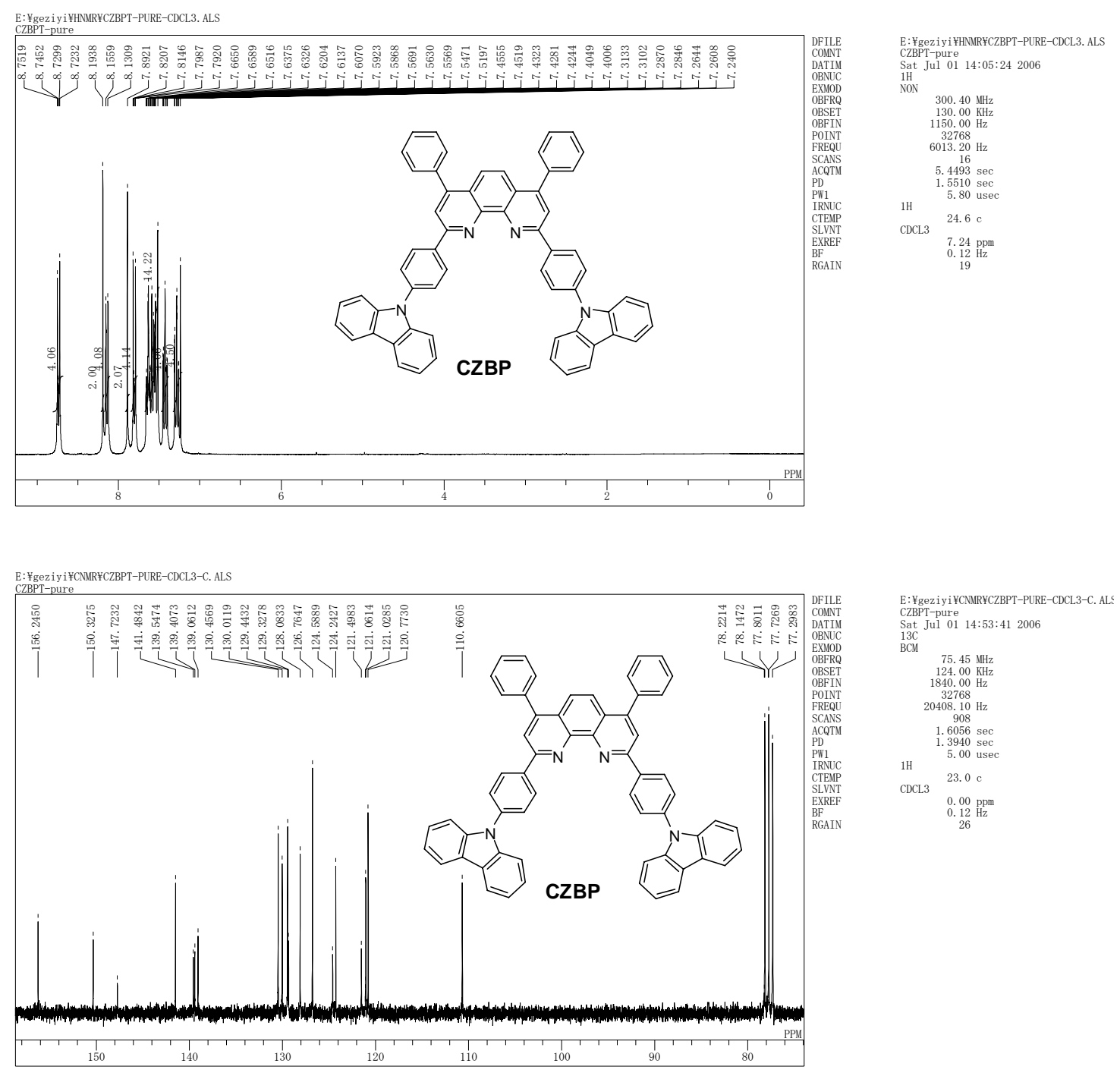


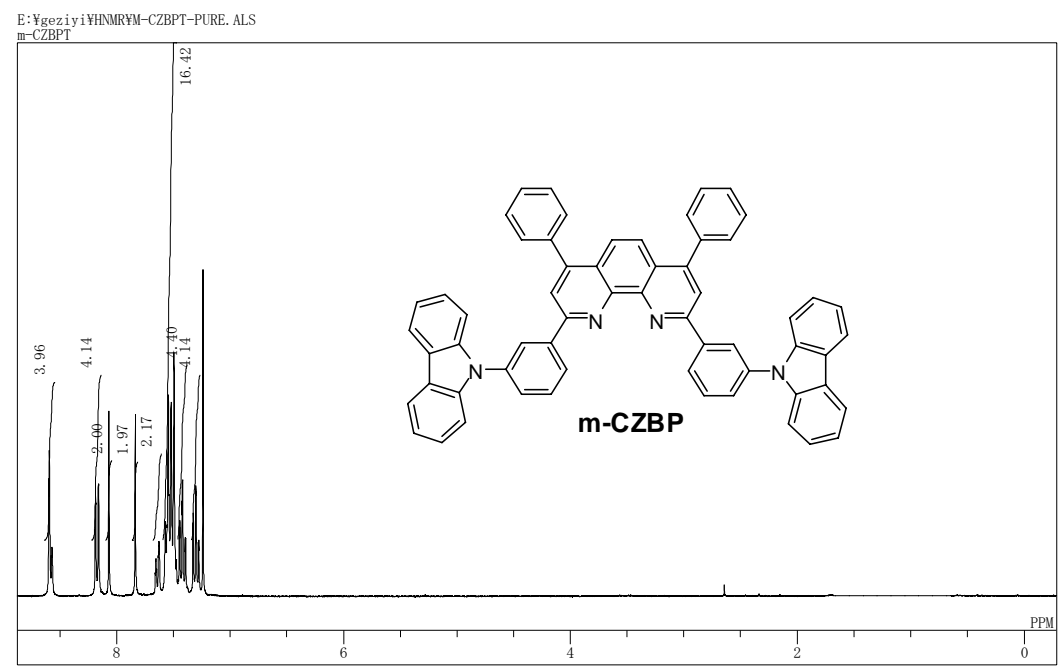

E: Ygezi yi YHNMR¥M-CZBPT-PURE. ALS

m-CZBPT
Thu Aug 17 16:01:41 2006
$1 \mathrm{H}$

$1 \mathrm{H}$

300. $40 \mathrm{MHz}$

$1150.00 \mathrm{H} z$

32768
$6013.20 \mathrm{~Hz}$

$5.4493 \mathrm{sec}$
$1.5510 \mathrm{sec}$

1H. $\quad 50$ use

CLCL3

$23.9 \mathrm{c}$

RGAIN

$7.24 \mathrm{ppm}$
$0.12 \mathrm{~Hz}$
2.1

E: :Ygezi iYYCCMRYM-CZBPT-C. ALS

Th-CZBPT 17 16:39:45 2006

${ }_{13 \mathrm{C}}^{13}$

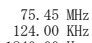

32768
20408.10

$1.6056 \mathrm{sec}$
$1.3940 \mathrm{sec}$

5.00 usec

CDCL3 $24.1 \mathrm{c}$

$0.00 \mathrm{ppm}$
$0.12 \mathrm{~Hz}$
26

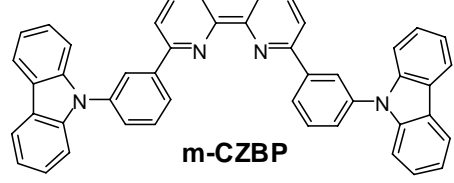

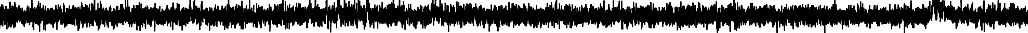
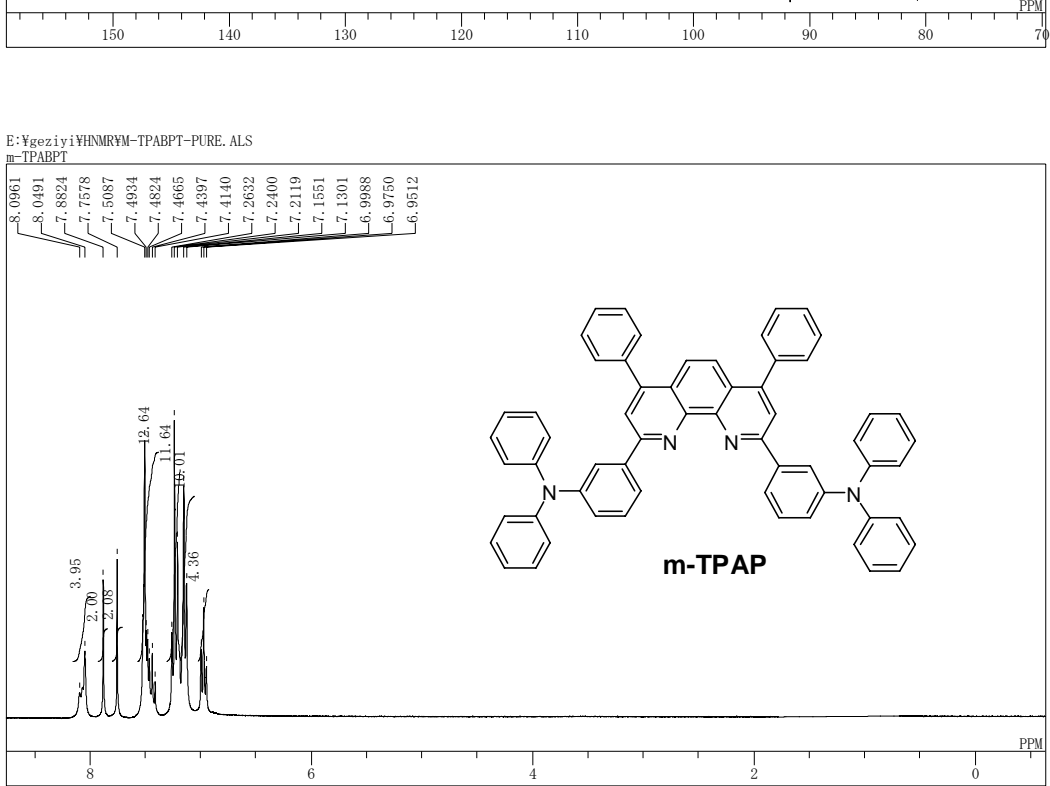

E: Y.Yezi yi YHNMRYM-TPABPT-PURE. ALS
m-TPABPT

Wed Sep 06 11:25:59 2006
1H

300. $40 \mathrm{MHz}$
$130.00 \mathrm{KHz}$

$1150.00 \mathrm{~Hz}$

6013. $20 \mathrm{~Hz}$

$5.4493 \mathrm{sec}$
$1.5510 \mathrm{sec}$

$1.5510 \mathrm{sec}$
$5.80 \mathrm{usec}$

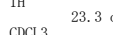

$\begin{array}{ll}\text { CDCL3 } & 7.24 \mathrm{ppm} \\ & 0.12 \mathrm{~Hz}\end{array}$ 


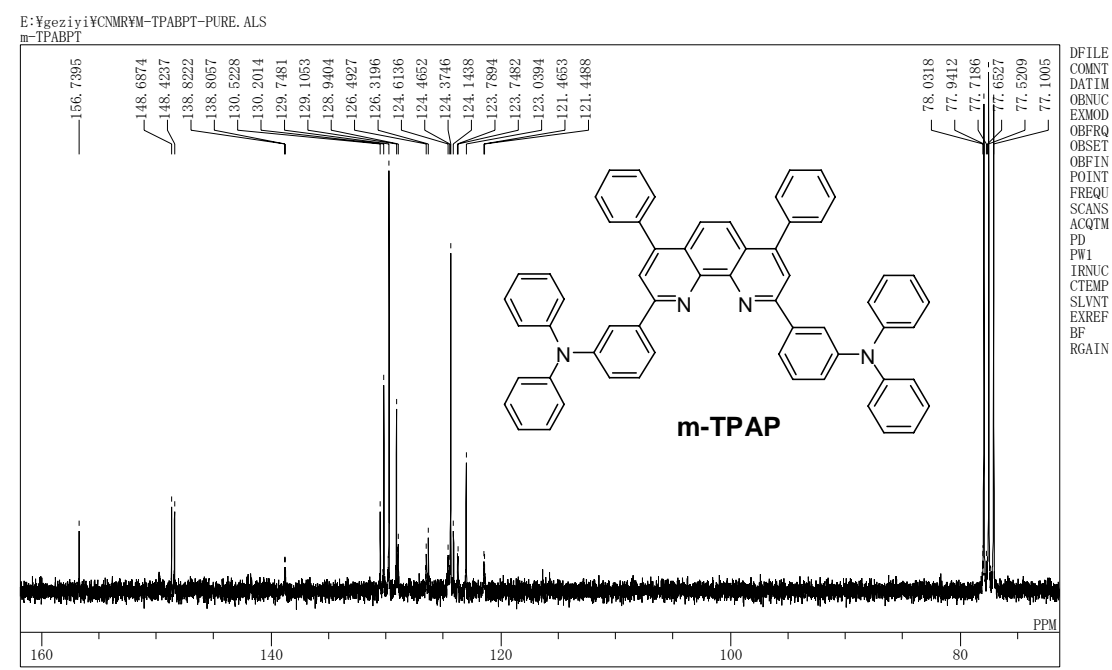

E: Ұgez i i i YCNMRYM-TPABPT-PURE. ALS

m-TPABPT
Wed Sep 06 12:19:53 2006
13C

$13 \mathrm{C}$
$\mathrm{BCM}$

$75.45 \mathrm{MHz}$
$124.00 \mathrm{KHz}$

${ }_{32768}^{1840.00 \mathrm{H}}$

32768
$20408.10 \mathrm{~Hz}$
1024

1. $6056 \mathrm{sec}$
1. $3940 \mathrm{sec}$

$1 \mathrm{H} \quad 5.00$ usec

CDCL3

$23.4 \mathrm{c}$

$0.00 \mathrm{ppm}$
0.12
24

E: Ygezi yi YHNMRYBP-TPABPT. ALS
BP-TPABPT

Fri Oct 13 09:31:31 2006

IH

300. $40 \mathrm{MHz}$
$130.00 \mathrm{KHz}$

${ }_{32768}^{1150.00 \mathrm{H}}$

6013. $20 \mathrm{~Hz}$

5. $4493 \mathrm{sec}$
$1.5510 \mathrm{sec}$

$1 \mathrm{H} \quad 5.80 \mathrm{usec}$

$\operatorname{CDCl}$

$7.24 \mathrm{ppm}$
$0.12 \mathrm{~Hz}$
18

E: Ұgezi yi i

Sun 0ct 15 10:02:28 2006

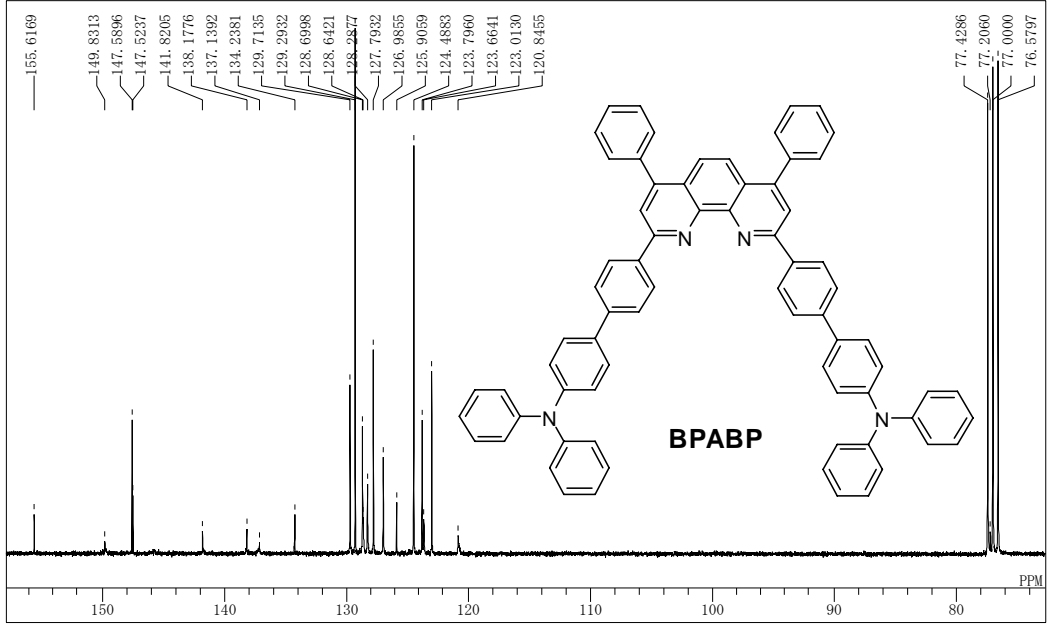

